\title{
Improving Diabetes Care by Data Feedback and Diabetes Templates
}

\author{
Charles M. Sow ${ }^{1}$, Folashade S. Omole1, Gregory Strayhorn1, Dominic Mack², \\ William A. Davis ${ }^{1}$, Olabisi Omole ${ }^{1}$ \\ ${ }^{1}$ Department of Family Medicine, Morehouse School of Medicine, East Point, USA \\ ${ }^{2}$ National Center for Primary Care (NCPC), Morehouse School of Medicine, Atlanta, USA \\ Email: csow@msm.edu, fomole@msm.edu, gstrayhorn@msm.edu, dmack@msm.edu, wdavis@msm.edu, \\ olabisiomole@gmail.com
}

Received 11 September 2014; revised 16 October 2014; accepted 5 November 2014

Copyright (C) 2014 by authors and Scientific Research Publishing Inc.

This work is licensed under the Creative Commons Attribution International License (CC BY). http://creativecommons.org/licenses/by/4.0/

(c) (i) Open Access

\begin{abstract}
Importance: Health information technology has been used to improve diabetes care and outcomes. With the implementation of our diabetes registry, we discovered several flaws in the data. Objective: The aim of this paper is to demonstrate whether improving diabetes templates in electronic medical records associated with data feedback, improves process and quality outcomes for patients with diabetes. Methods: We redesigned our chronic diseases templates and clinical flow, built a diabetes registry and used the data for feedback to educate providers, staff and address inconsistencies. A total of 724 active diabetic patients were identified in October 2009 (pre-implementation) and 731 active diabetic patients were identified in June 2011 (post-implementation). Results: The results showed an improvement in the process outcomes of ordering hemoglobin A1C every 6 months and a microalbumin every 12 months (p-value $<0.05$ ), but no change in the quality outcomes hemoglobin A1C less than 7, blood pressure less than 140/90 and LDL cholesterol less than 100 (p-value > 0.05). Discussion: Data feedback and lessons learned were instrumental to our practice change.
\end{abstract}

\section{Keywords}

Electronic Health Records, Diabetes Registry, Data Feedback

\section{Introduction}

Crossing the Quality Chasm (A New Health System for the 21st Century), found that the nation's health care industry had fallen short in its ability to provide safe, high-quality care consistently to all Americans, but partic-

${ }^{*}$ Corresponding author.

How to cite this paper: Sow, C.M., Omole, F.S., Strayhorn, G., Mack, D., Davis, W.A. and Omole, O. (2014) Improving Diabetes Care by Data Feedback and Diabetes Templates. Open Journal of Preventive Medicine, 4, 829-833.

http://dx.doi.org/10.4236/ojpm.2014.411093 
ularly to those with chronic conditions [1].

Dr. Ed Wagner and the Improving Chronic Illness Care (ICIC), described that the aim of the Chronic Care Model was to improve patient health outcomes by changing the routine delivery of ambulatory care for patients with chronic illnesses from acute and reactive to proactive, planned, and population-based [2]. Morehouse Family Medicine Residency Program received a Health Resources and Services Administration (HRSA) residency training grant to develop a Chronic Care Model curriculum with a focus on diabetes [2] [3]. One of the goals was to develop a diabetes registry to improve clinical outcomes. We discovered several flaws in the data with the implementation of the diabetes registry.

Previous studies utilizing health information technology showed improvement in diabetes outcomes [4] [5].

The aim of this paper is to demonstrate improved process and quality outcomes for patients with diabetes by redesigning our healthcare delivery system with data feedback.

\section{Methods}

This quality improvement project was approved by Morehouse School of Medicine Institutional Review Board and had determined the procedures appropriate for exemption under the federal regulations.

Study site: The Comprehensive Family Health Center (CFHC) is one of the family medicine residency training clinical sites with over 20,500 encounters per year. The CFHC is located south of Atlanta in the tri-city locale of East Point, College Park and Hapeville. South Atlanta is an underserved area, and is comprised predominantly of African American and Hispanic patients. Many of our patients come from under-privileged and under-represented minority communities, who tend to have difficulty with access to needed care, and are less likely to receive routine care and more likely to be seen for episodic/acute care. The socioeconomic scale ranges from indigent to upper middle income. The patient population is skewed towards African-Americans, young to middle aged adult females, and those with a high school education; with mixed insurance coverage.

The residency program has 18 residents and 10 faculty members. We used practice partner electronic health records (EHR) from McKesson; all diabetes templates were from the vendor and were not customized to our practice.

\subsection{Participants}

Patients were all identified as diabetes based on ICD-9 codes of diabetes and age over 18. Patients were defined as active in the practice if they have had a visit within 1 year and are not designated as deceased, demonstration, transferred, or inactive status. Diagnoses are active if they have been entered within the past 3 years. Patients with more than one year chart inactivity were excluded from the review.

In July 1, 2001 with funds from the Health Resources and Services Administration (HRSA), an agency of the US Department of Health and Human Services HRSA, we adopted the Electronic Health Records (EHR). All staff, faculty and residents were trained to use the EHR. All paper charts were removed from the practice after one year of the EHR implementation. On July 1, 2007, with additional HRSA funding, we developed and began the implementation of the chronic care model (CCM) with an initial focus on Diabetes. In collaboration with PPRNet (Primary (Care) Practices Research network), and Recombinant Data, Inc., a data warehousing company specializing in the secondary use of clinical data, we set out to create a diabetes patient registry; practice and individual practitioner reports to identify opportunities for intervention in diabetic care and measure progress on a variety of quality metrics from the National Committee for Quality Assurance (NCQA) Diabetes Physician Recognition Program (DPRP) [6].

\subsection{Pre-Intervention Issues}

We had too many non-functional templates for one disease and not user friendly. There was an over reliance on free text. Heath Maintenance tables and templates were not updated. Scanned referral reports did not update Health Maintenance tables. Reminder alert for preventive services was off. There was an inconsistency with laboratory data with occasional interface of laboratory data with EHR laboratory tables resulting in numerous missing lab values.

\subsection{Intervention}

We redesigned the chronic disease templates (diabetes, hypertension, and hyperlipidemia) by developing new, 
user-friendly templates, creating auto-populating templates. The non-functional templates were discarded. The reminders for preventive services were turned on. The cross reference code of the laboratory data was updated to ensure proper interface process; and reloaded all missing old laboratory data.

Using the data warehouse we created three diabetes quality improvement reports: the provider, the patient, and the Diabetes Physician Recognition Program (DPRP) report which compared providers' performance with their peers. Providers and patients received report cards documenting individual patient blood pressure, hemoglobin A1C levels, cholesterol, medications and treatment plans.

1) Creation of new diabetes templates: the diabetes templates automatically pulled the date and value of last hemoglobin A1C, last lipid panel, micro-albumin, vitals of current visit, last foot exam, last dilated eye exam and immunizations (influenza and pneumonia). This is contrary to the old template where providers had to go to several tabs to review all the information in regards to diabetes care.

2) Health Maintenance tables were auto-populated from scanning reports. We redesigned the vitals templates with uniformity in vitals entry by medical assistants and nurses.

3) Creation of a patient diabetes report card: all identified diabetic patients received a self-management report card based on American Diabetes Association guidelines at each visit and results were discussed with their providers. The report card included the date and value of the last hemoglobin A1C, lipid panel, and micro-albumin, last dilated eye and foot exams, immunizations (influenza and pneumonia).

4) Creation of a diabetes registry based on NCQA's Diabetes Physician Recognition Program (DPRP). In collaboration with our Clinical Research Center at Morehouse School of Medicine, we started building our clinical data warehouse. Data cleaning algorithms were designed through close collaboration between clinicians and technical personnel. Additional algorithms were developed to convert unstructured data to selected discrete data elements in the data warehouse. During this phase we also corrected inconsistencies in metric regarding weight and height (e.g. pounds vs. kilograms, inches vs. centimeters), allowing historical data to be realigned with the new standards, and providers focusing on ensuring the accuracy of new data. While the data warehouse retains historical data, its data cleaning algorithms and reference ranges ensure that incorrect data does not appear in reports.

5) We started the diabetes group visits to educate our patients about diabetes and chronic diseases management. The group visit was opened to patients and their families and was conducted by a faculty, a resident and a dietician.

\subsection{Statistical Analysis}

We compared our data pre- and post-intervention, using our diabetes data from October 2009 (pre-implementation) and June 2011 (post-implementation). We measured and compared the diabetes outcome processes: diabetic patients with blood pressure measured in the past 6 months, hemoglobin A1C measured in the past 6 months, LDL cholesterol measured in the past 12 months and urine microalbumin measured in the past 12 months.

We measured and compared the diabetes quality outcomes: diabetic patients with most recent hemoglobin A1C less than 7\%, most recent LDL cholesterol less than 100 and most recent blood pressure less than 140/90. We used frequency, proportions, chi-square and Fischer's exact test to analyze the data.

\section{Results}

A total of 724 active diabetic patients were identified in October 2009 (pre-implementation) and 731 active diabetic patients were identified in June 2011(post-implementation) with their characteristics as showed in Table 1. Process outcomes with blood pressure measured in the past 6 months (p-value 0.77) and LDL cholesterol measured in past 12 months (p-value 0.63 ) did not show any change in contrary to hemoglobin A1C in past 6 months (p-value 0.02) and urine microalbumin in the past 6 months (p-value 0.01) showed statistically significant improvement (Table 2). Quality outcomes as blood pressure less than 140/90 (p-value 0.17), most recent hemoglobin A1C less than 7\% (p-value 0.10), most recent LDL cholesterol less than 100 (p-value 0.64) did not show any improvement (Table 3).

\section{Discussion}

These results showed an improvement in the process outcomes of ordering hemoglobin A1C every 6 months and 
Table 1. Demographics.

\begin{tabular}{ccc}
\hline & Pre-implementation & Post-implementation \\
\hline Female & $455(62.8 \%)$ & $473(64.7 \%)$ \\
Male & $269(37.2 \%)$ & $258(35.3 \%)$ \\
Mean age & 59.82 & 60.81 \\
\hline
\end{tabular}

Table 2. Process outcomes.

\begin{tabular}{lccc}
\hline & Pre-implementation & Post-implementation & p-value \\
\hline Diabetics with blood pressure measured in past 6 months & 564 (78\%) & 574 (79\%) & 0.77 \\
Diabetic patients with hemoglobin A1C measured in past 6 months & $365(50 \%)$ & $413(57 \%)$ & 0.02 \\
Diabetic patients with LDL cholesterol measured in past 12 months & $531(73 \%)$ & $528(72 \%)$ & 0.63 \\
Diabetic patients with urinary microalbumin measured in past 12 months & $333(46 \%)$ & $382(52 \%)$ & 0.01 \\
\hline
\end{tabular}

Table 3. Quality outcomes.

\begin{tabular}{|c|c|c|c|}
\hline & Pre-implementation & Post-implementation & p-value \\
\hline Diabetic patients with most blood pressure $<140 / 90$ & $329(58 \%)$ & $312(54 \%)$ & 0.17 \\
\hline Diabetic patients with most recent hemoglobin A1C $<7 \%$ & $165(45 \%)$ & $211(51 \%)$ & 0.10 \\
\hline Diabetic patients with most recent LDL cholesterol $<100$ & $294(55 \%)$ & 285 (54\%) & 0.64 \\
\hline
\end{tabular}

a urine microalbumin every 12 months (p-value $<0.05$ ). These processes were automated by printing a diabetes report for the patient that included the last $\mathrm{A} 1 \mathrm{C}$ and microalbumin in the progress note. The provider intervention to place orders was facilitated by data directly accessible from the diabetes template. Blood pressure did not change since it was incorporated in the clinical flow process that all patients needed vitals taken at each encounter.

The implementation of our EHR did not correspond to better quality outcomes in our diabetes care. We can find several explanations for the lack of improvement, the results of the lab were not available at the time of visit but one or two days after the visit and follow-up was needed with a phone call to address issues with uncontrolled diabetes.

Improper patient encounter documentation using EHR has an impact on quality of care provided to the patient. Implementing the EHR turned out to be just the first step in improving patient care. Effective reporting with high quality data is a significant contributor to the meaningful use of the EHR, which consequently helps patients and providers understand which activities are improving the quality of care and driving outcomes. The impact of EHR reporting can be seen in our pre- and post-implementation reporting of DPRP measures. Use of achievable benchmarks significantly enhances the effectiveness of physician performance feedback [7]. The introduction of the first diabetes report was instrumental to the cultural change. In collaboration with the GO (Georgia Oklahoma) Diabetes Project [8], our residents had several projects, including educational programs for providers such as "improving diabetic patient referral for annual eye exams" and "improving annual comprehensive foot exams", among many. Data must be perceived by physicians as valid to motivate change [9]. A major barrier to overcome is physicians' trying to exclude non-compliant patients from their panels because of fear of penalty as a result of their report card. The engagement of all providers in the entire process of transformation and the focus on data quality, helped physicians overcome this fear and to regard the reports as a mechanism to help them achieve goals that they all support in principle.

Lessons learned from the diabetes patient and provider report cards were instrumental in our quality improvement. However, quality diabetes process indicators of providers which are time-based, in part depend upon patient encounters. In order to remove this barrier, we will implement a health coach to assure appointments are made and kept. Quality diabetes outcome indicators for patients were considerably lower than quality process indicators. Thus, it was necessary to target patient behaviors which influence these indicators through patient education and diabetes group visits. Our diabetes quality improvement project laid the groundwork for additional improvements in other chronic diseases and preventive care. 


\section{Study Limitations}

Our study was retrospective pre- and post-implementation by analyzing the data from our diabetes registry. We did not control for the population at baseline and follow-up. A randomized controlled study will be better with follow-up of the same population.

\section{Acknowledgements}

HRSA grant \# D58HP08294. The final manuscript has been seen and approved by all the authors. There are no conflicts of interest and the authors have nothing to disclose.

\section{References}

[1] IOM (2001) Crossing the Quality Chasm: A New Health System for the 21st Century. http://books.nap.edu/openbook.php?record_id=10027\&page=R1\#

[2] (2014) Improving Chronic Illness Care: The Chronic Care Model. http://www.improvingchroniccare.org/change/index.html

[3] Wagner, E.H. (1998) Chronic Disease Management: What Will It Take to Improve Care for Chronic Illness? Effective Clinical Practice, 1, 2-4.

[4] Hunt, J.S., Siemienczuk, J., Gillanders, W., et al. (2009) The Impact of a Physician-Directed Health Information Technology System on Diabetes Outcomes in Primary Care: A Pre- and Post-Implementation Study. Informatics in Primary Care, 17, 165-174.

[5] O’Connor, P.J., Crain, A.L., Rush, W.A., Sperl-Hillen, J.M., Gutenkauf, J.J. and Duncan, J.E. (2005) Impact of an Electronic Medical Record on Diabetes Quality of Care. Annals of Family Medicine, 3, 300-306. http://dx.doi.org/10.1370/afm.327

[6] NCQA (2014) Diabetes Recognition Program 2009. http://www.ncqa.org/tabid/139/default.aspx

[7] Kiefe, C.I., Allison, J.J., Williams, O.D., Person, S.D., Weaver, M.T. and Weissman, N.W. (2001) Improving Quality Improvement Using Achievable Benchmarks for Physician Feedback. JAMA: The Journal of the American Medical Association, 285, 2871-2879. http://dx.doi.org/10.1001/jama.285.22.2871

[8] Berryman, S.H., Sick, B.T., Wang, Q., Swan, P.J. and Weber-Main, A.M. (2013) Use of Automated Reminder Letters to Improve Diabetes Management in Primary Care: Outcomes of a Quality Improvement Initiative. Quality in Primary Care, 21, 359-368.

[9] Bradley, E.H., Holmboe, E.S., Mattera, J.A., Roumanis, S.A., Radford, M.J. and Krumholz, H.M. (2004) Data Feedback Efforts in Quality Improvement: Lessons Learned from US Hospitals. Quality and Safety in Health Care, 13, 26-31. http://dx.doi.org/10.1136/qhc.13.1.26

\section{Abbreviations}

EHR: Electronic Health Records

CCM: Chronic Care Model

DPRP: Diabetes Physician Recognition Program

HRSA: Health Resources and Services Administration

NCQA: National Committee for Quality Assurance

PPRNet: Primary (Care) Practices Research Network 
Scientific Research Publishing (SCIRP) is one of the largest Open Access journal publishers. It is currently publishing more than 200 open access, online, peer-reviewed journals covering a wide range of academic disciplines. SCIRP serves the worldwide academic communities and contributes to the progress and application of science with its publication.

Other selected journals from SCIRP are listed as below. Submit your manuscript to us via either submit@scirp.org or Online Submission Portal.
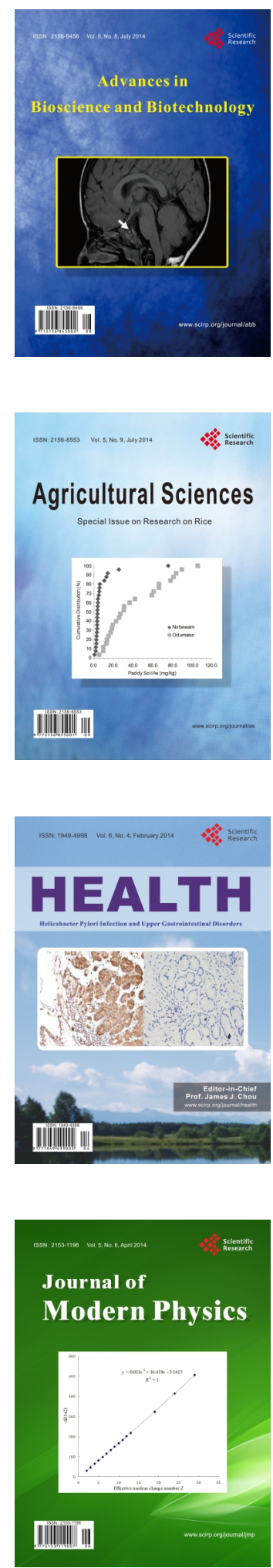
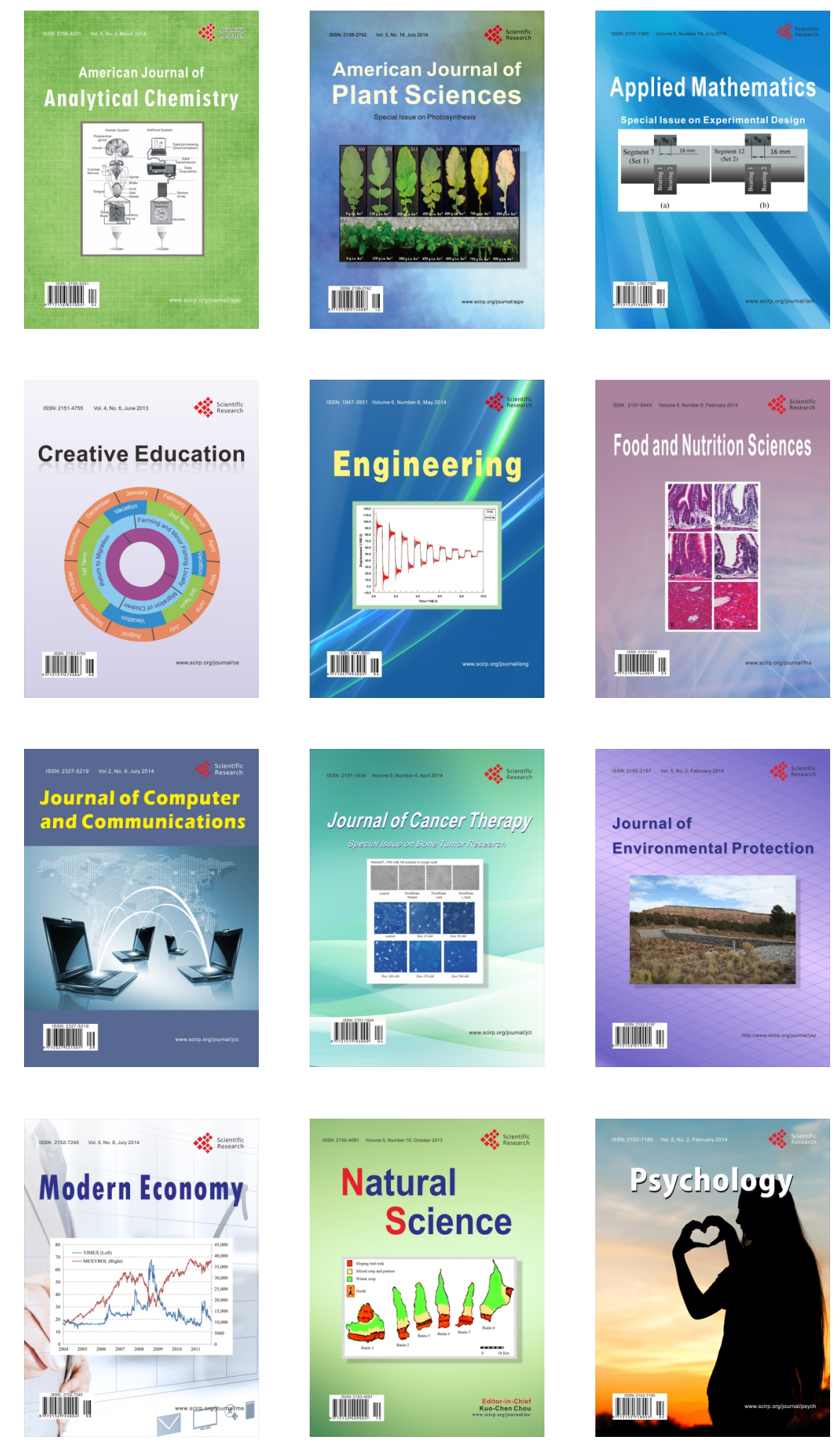